Original Research

\title{
Identifying Half-Century Precipitation Trends in a Chinese Lake Basin
}

\author{
Muhammad Tayyab ${ }^{1,2}$, Xiaohua Dong ${ }^{1,6 *}$, Ijaz Ahmad ${ }^{3}$, Aqeela Zahra ${ }^{4}$, \\ Jianzhong Zhou' ${ }^{2}$ Xiaofan Zeng ${ }^{2}$, Aamir Shakoor ${ }^{5}$
}

${ }^{1}$ College of Hydraulic and Environmental Engineering, China, Three Gorges University, Yichang, China ${ }^{2}$ School of Hydropower and Information Engineering, Huazhong University of Science and Technology, Wuhan, China ${ }^{3}$ Centre of Excellence in Water Resources Engineering, University of Engineering and Technology, Lahore, Pakistan ${ }^{4}$ School of Life Sciences, South China Normal University, Guangzhou, China

${ }^{5}$ Department of Agricultural Engineering, Bahauddin Zakariya University, Bosan Road, Multan, Pakistan ${ }^{6}$ Hubei Provincial Collaborative Innovation Center for Water Security, Wuhan, 430070, China

Received: 3 December 2017

Accepted: 22 February 2018

\begin{abstract}
This research investigates the spatial and temporal trend analysis of precipitation time series. Precise predictions of precipitation trends can play an imperative role in economic growth of a country. This study examined precipitation inconsistency for 23 stations at Dongting Lake, China, over a 52-year study period (1961-2012). Statistical, nonparametric Mann- Kendall (MK) and Spearman's rho tests were applied to identify trends within monthly, seasonal, and annual precipitation. The trend-free pre-whitening method was used to exclude sequential correlation in the precipitation time series. The performance of the Mann-Kendall (MK) and Spearman's rho tests was steady at the tested significance levels. The results showed a fusion of increasing and decreasing trends at different stations within monthly and seasonal time scales. The results obtained with the Mann-Kendall and Spearman's rho tests showed agreement in their assessments of monthly, seasonal, and annual precipitation trends. The variability of negative and positive trends at various stations points to the need for more detailed studies on the climate change of this region. In the case of whole Dongting basin on the monthly time scale, a significant positive trend is found, while at Yuanjiang River and Xianjiag River both positive and negative significant trends are identified. Only Yuanjiang River has shown a significant trend on the seasonal time scale. No significant trends have been exhibited on the annual time scale in any case. In the case of monthly, Nanxian station exhibited the maximum positive increase in monthly precipitation during the months of July and September. In the case of seasonal, only Tongren station showed a positive trend on the monthly level, and no significant negative trends were detected in both spring and autumn seasons.
\end{abstract}

Keywords: Mann-Kendall (MK), Spearman's rho, Dongting Lake, increasing trend, decreasing trend

*e-mail: xhdong@ctgu.edu.cn 


\section{Introduction}

The discovery of variations in precipitation is a vital and hard issue that is of growing interest because of its central role in the development of future water resources and flood safety. Numerous statistical tests and dynamic approaches have been established to detect trends in hydro-meteorological and hydrological time series, categorized as parametric and nonparametric tests [1]. Parametric tests stand dominant but data should be normally distributed and independent or autonomous, which is hardly the case in hydrological time series data. On the other side, nonparametric test data should be independent, but other conditions can be better tolerated. Scientists have found that the most common nonparametric tests are the Mann-Kendall [2], Spearman's rho, and Theil Sen's [3] for investigating the trends in time series data. The Mann-Kendall test is the most commonly applied by researchers in studying hydrologic time series trends $[4,5]$; less common, Theil Sen's and Spearman's rho are used to detect magnitude and monotonic trends in hydro-meteorological data, respectively [6]. In several types of research, Spearman's rho is used as the combination with the Mann-Kendall test for evaluation purposes [7-9].

Dongting Lake, the largest interior lake in China, is located in the middle Yangtze River region. Over the past decades, Dongting has experienced intensified human activities. The lake margins have been reclaimed heavily for agriculture, and deforestation has prevailed in its upper catchment and adjacent area [10]. It is anticipated that Dongting will continue to shrink, and in the near future may serve only as a river channel during no-flood conditions. However, the construction of large hydraulic works such as the Three Gorges Dam may help decelerate lake shrinkage, as a large fraction of sediment will be trapped behind the dam [11]. The complexity of this background gives added significance to studies of the middle Yangtze region. For example, Chen et al. [12] analyzed the trends on precipitation time series in the Yangtze River Basin by using precipitation data together with discharge data. The spatial-temporal variability of precipitation during the last half century in the Yangtze River Basin was analyzed and the TrendFree Pre-Whitening (TFPW), Mann-Kendall (MK) statistical test was applied to assess the significance of trends [13]. Wang and Zhou [14] examined the longterm trend and periodic variation of temperatures and precipitation in the Chuanjiang Section of the Yangtze River were analyzed using both parametric and nonparametric methods. The possible association between climate change trend over the Tibetan Plateau and the variability of water resources in the upper reaches of the Yangtze River was then investigated [15].

Most previous studies have focused on regional climate change in the Yangtze River Basin or its upper reaches. However, a few studies have been done on the climate tendencies in the middle Yangtze River, except for trend analysis variation mainstream of the middle
Yangtze River [16]. There has been increasing concern about the threat of global warming in the past decade. Global climate warming and its impacts have been a key research area during recent decades. According to the Fourth Assessment Report (AR4) from the Intergovernmental Panel on Climate Change (IPCC), observational evidence for all continents and most oceans show that many natural systems are being affected by regional climate change, particularly by the increase of air temperature and precipitation [17]. The annual and seasonal trends of seven meteorological variables were analyzed for twelve weather stations in Serbia during 1980-2010. The non-parametric Mann-Kendall and Sen's methods were used to determine whether there was a positive or negative trend in weather data with their statistical significance. In general, the results of using the Mann-Kendall and Sen's tests demonstrated the good agreement of performance in detection of the trend for meteorological variables [18]. Numerous researchers have led a large number of investigations around the world to notice the climate change and carrying out trend analysis. Yunling and Yiping [19] analyzed the trends in climatological variables in China beside the Lancer River for 19 stations between 1960 and 2000. Gu et al. [20] applied the Mann-Kendall test for detecting the runoff trend in major river basins of China, and the results have shown a great deal of accuracy. Fan and Wang [21] calculated temperature trend by applying the Mann-Kendall method in Shanxi Province in China and found an increasing temperature trend. Jiang et al. [22] examined wind speed trend during 1956-2004 in China by applying Mann-Kendall. They used two datasets and found that the trend is decreasing in the area. Most of these studies concentrated on regional precipitation fluctuations in the Yangtze River Basin.

An increasing annual precipitation trend was found by using Mann-Kendall and Sen's slope tests in the Uttrakhand region of India from 1901-2010 [23]. Investigating precipitation trends is carried out by applying Mann-Kendall (MK) and Spearman rho (SR) tests for detecting the trend on the seasonal and annual basis at 5\% significance level for the time period of 51 years (1961-2011) in the Swat River Basin of Pakistan. The performance of MK and SR tests was found to be consistent in detecting the trend at different stations [24]. On the western part of the French Mediterranean area, namely the Pyrénées-Orientales and Aude, the non-parametric MK test was applied to identify significant trends at the local scale and, because of the natural spatial variability of the Mediterranean climate, regional interpretation was also performed. The regional annual and seasonal precipitation trends at the Langat River Basin, Malaysia, for the period of 1982-2011 were examined using MK. From the annual scale perspective, all three regions showed positive trends $[25,26]$. MK was applied to identify existing trends in annual, seasonal, and monthly rainfall at thirteen stations in the Onkaparinga catchment in South Australia during the period 1960-2010. Most of the trends, whether positive 
or negative, started during the mid-1970s to mid-1980s [27]. The Mann-Kendall non-parametric Spearman correlation coefficient (rho) was used to determine the interannual variability in precipitation trends in central Finland during the period 1959-2009. The performance of MK and SR tests was consistent in detecting the trend at different stations [28]. The homogeneity analysis of temporal (monthly, seasonal, and annual) climate aridity index trend was accomplished for 43 climate measurement stations in South Korea by applying MK. The study results showed the temporal and regional homogeneity of climate aridity index trends for individual and entire 9 regions [29].

In further studies, Singh et al. [30] used MK to detect precipitation trend and relative humidity in India, and have found an increasing trend in relative humidity in most parts of the river basin. Sequential MK technique is becoming popular for determining the start of a trend and abrupt change in trends [31, 32]. The objectives of this study were to investigate trends in annual precipitation, sunshine duration, wind speed (u 2), and annual and monthly minimum temperatures ( $\mathrm{T}$ min), maximum temperature ( $\mathrm{T} \max$ ), and relative humidity $(\mathrm{RH})$, and the adaptation strategies for the Senegal River Basin. Annual precipitation, T min, T max, RH, sunshine duration, and u 2 for the period of 1950-2000 recorded at St. Louis, Bakel, Dagana, Fanaye, Podor, and Matam have been analysed using MK and Sen's slope estimator [33], which were employed to determine precipitation trends at 48 stations located in the core of the North American monsoon. The results disclose a significant upward trend in the intensity of P95 increases in mountainous stations, suggesting a greater contribution of precipitation associated with TCs [34, 35]. Spectral analysis of Lake Urmia level time series and impact of climate and hydrological variables on it using MK also has shown a great deal of applicability [36]. By using the MK method, the effects of short- and long-term persistence on identifying temporal trends were examined by [37]. By using MK streamflow trends and climate variability, the impacts in Poyang Lake basin have been analyzed by Zhao et al. [38], who found that the nonparametric tests have a tendency to produce better results. At Urmia Lake Iran, three types of nonparametric test have been used and all of these tests have shown a great deal of accuracy [39]. Gridded rainfall data of $0.5 \times 0.5^{\circ}$ resolution (CRU TS 3.21) was analyzed to study long-term spatial and temporal trends on annual and seasonal scales in Wainganga river basin located in central India during 1901-2012. Theil and Sen's slope estimator test was used for finding the magnitude of change over a time period. Though most of the grid points show a decreasing trend in annual rainfall, only seven grids show a significant decreasing trend during 1901-2012 [40].

The present study examined monthly, seasonal, and annual precipitation from 23 main meteorological stations during the period of 1961-2012 in Dongting Lake. The short-term trends of monthly and long-term trends of precipitation on seasonal and annual time scale were analyzed in Dongting Lake. At the same time, the characteristics of precipitation trends at each sub-basin as well as the entire basin were investigated for the first time in this paper. The outcomes of this research may possibly deliver significant evidence for the preparation of upcoming water resources and flood safety mechanisms in Dongting Lake.

\section{Material and Methods}

\section{Study Area}

This study investigates the variability in precipitation time series for a 52-year period (1961-2012) in Dongting Lake (Fig. 1). Dongting is situated on the south shoreline

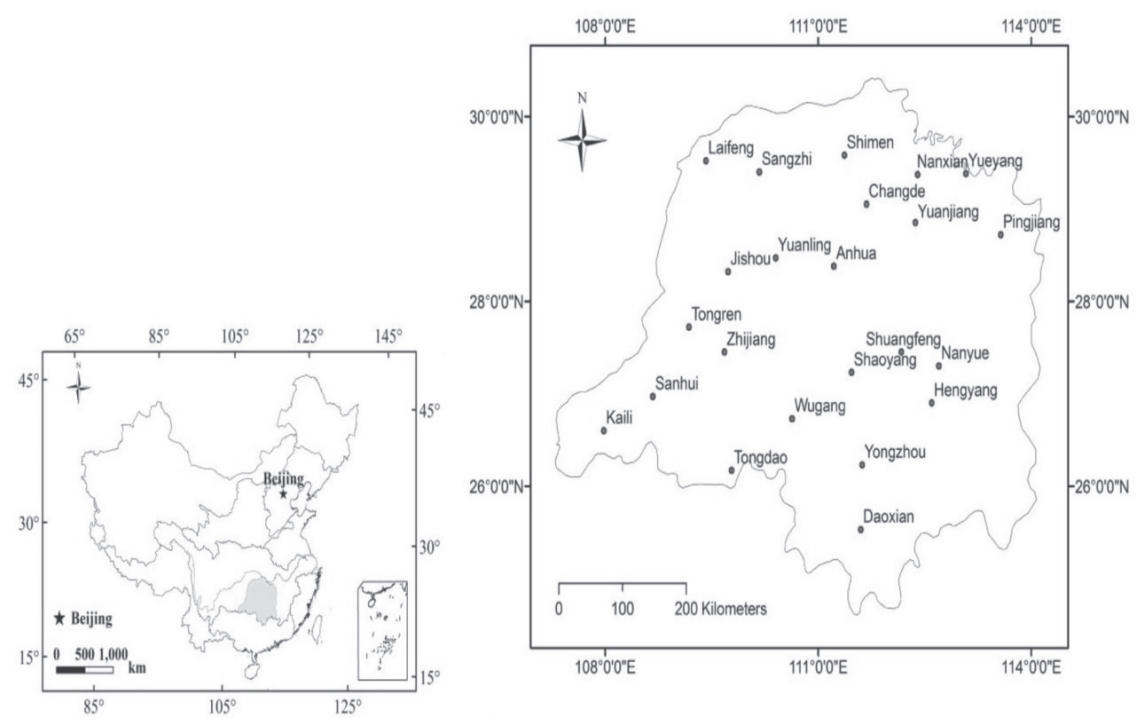

Fig. 1. Locations of meteorological stations at Dongting Lake. 
Table 1. Different seasons for analysis.

\begin{tabular}{|c|c|c|}
\hline Sr. number & Season & Month \\
\hline 1 & Anually & Jan-Dec \\
\hline 2 & Winter & Dec-Feb \\
\hline 3 & Spring & Mar-May \\
\hline 4 & Summer & Jun-Aug \\
\hline 5 & Autumn & Sep-Nov \\
\hline
\end{tabular}

of the middle ranges of the Yangtze River. The lake observed a frequent progression procedure beginning from small to big and big to small, which is thoroughly connected to the evolution of the Jingjiang network. The inflow of Dongting Lake largely comes from the Yangtze. Since 1948, numerous structural developments have been upsetting the state of the water flowing to the lake, primarily the assembly of Tiaoxian Dam, the project of Jingjiang and Wanjiangfengxi Reservoir, Zishuituoxi Reservoir, Gezhouba Dam, and the Three Gorges Reservoir.

\section{Data and Method}

The total Dongting catchment area is $262,108 \mathrm{~km}^{2}$, which is about $12 \%$ of the total Yangtze River, having 23 meteorological stations. It covers Hubei and Hunan provinces in the administrative division. The important branches include Lake Region (A1), Lishui River (A2), Yuanjiang River (A3), Zishui River (A4), and Xiangjiang River (A5). The catchment areas for these basins are $27965 \mathrm{~km}^{2}, 28328 \mathrm{~km}^{2}, 91319 \mathrm{~km}^{2}, 19669 \mathrm{~km}^{2}$, and $94826 \mathrm{~km}^{2}$, respectively. For the entire Dongting Basin (A), mean value of precipitation at the corresponding stations is used [41]. It also has a few small rivers, like the Xinqiang, Miluo, and additional smaller rivers. In the north, it contains four canals, i.e., Tiaoxian, Hudu Songzi, and Ouchi, and is linked to the Yangtze and putative water of the Yangtze River. Water and sediment from Dongting are dumped into the Yangtze at Chenglingji, which is situated at the passage of the lake. Precipitation data were also categorized into seasonal (winter, spring, summer, and autumn) and annual data series as given in Table 1.

\section{Statistical Tests}

Prior to applying MK and SR tests to identify precipitation trends over the time series from selected stations, data were tested conferring to test requirements. The trend-free pre-whitening (TFPW) method was used to remove serial or sequential correlations in the time series data. The scale of the slope in time series data was attained by using Sen's slope method. The statistical methods used are briefly discussed below [42-46].

\section{Trend-Free Pre-Whitening Approach}

In the case of the non-parametric tests, the data set should be serially autonomous or independent. On the basis of previous studies, the presence of serial correlation will raise the prospect for important trend finding. That clues to an inconsistent refusal of the null hypothesis of no trend, while the null hypothesis is really true. Thus, the impact of serial correlation should be removed. For this purpose, few methods such as variance correction, pre-whitening [47], and TFPW [48] have been recommended. The TFPW method offered here delivers a good valuation of the significance of the trends for serially correlated data than the other methods. In this research, to eliminate the consequence of serial correlation on both tests (MK and SR) the TFPW technique was used for time series with significant autocorrelation coefficient $\left(r_{1}\right)$ at $5 \%$ significant level (see Eq. 1). The autocorrelation was tested against the null hypothesis by using Eq. 2:

$$
\begin{aligned}
& r_{1}=\frac{\sum_{i=1}^{n-1}\left(X_{i}-\bar{X}\right)\left(X_{i+1}-\bar{X}\right)}{\sum_{i=1}^{n}\left(X_{i}-\bar{X}\right)^{2}} \\
& r_{1}(95 \%)=\frac{-1 \pm 1.96 \sqrt{(n-2)}}{(n-1)}
\end{aligned}
$$

\section{The MK Test}

This is an overgrown statistical nonparametric test established by Mann-Kendall [49]. For this test, the null $\left(\mathrm{H}_{0}\right)$ and another hypotheses $\left(\mathrm{H}_{1}\right)$ are equivalent to the absence and is a trend in the time series of the present observational data, correspondingly. The associated equations to calculate the MK test statistic $\mathrm{S}$ and the consistent test statistic $\mathrm{Z}_{\mathrm{MK}}$ are as follows: mathematical equations 3 for calculating Mann-Kendall Statistics $(S)$, and standardized test statistics $Z$ are as follows:

$$
S=\sum_{i=1}^{n-1} \sum_{j=i+1}^{n} \operatorname{sgn}\left(X_{j}-X_{i}\right)
$$

$$
\operatorname{sgn}\left(X_{j}-X_{i}\right)=\left\{\begin{array}{c}
+1 \rightarrow \text { if }\left(X_{j}-X_{i}\right)>0 \\
0 \rightarrow \text { if }\left(X_{j}-X_{i}\right)=0 \\
-1 \rightarrow \text { if }\left(X_{j}-X_{i}\right)<0
\end{array}\right\}
$$

$$
\operatorname{Var}(S)=\frac{1}{18}\left[n(n-1)(2 n+5)-\sum_{p=1}^{n} t_{p}\left(t_{p}-1\right)\left(2 t_{p}+5\right)\right]
$$


Table 2. List of meteorological stations used in the DTL in this study.

\begin{tabular}{|c|c|c|c|c|c|c|c|c|c|}
\hline Station-ID & StationName & Latitude & Longitude & Altitude & Average mm & $\max$ & skew & kurt & std \\
\hline 57554 & Sangzhi & 29.40 & 110.17 & 322.20 & 117.94 & 671.70 & 1.80 & 4.09 & 105.85 \\
\hline 57574 & Nanxian & 29.37 & 112.40 & 36.00 & 104.10 & 614.30 & 1.50 & 3.71 & 80.27 \\
\hline 57584 & Yueyang & 29.38 & 113.08 & 53.00 & 109.79 & 571.60 & 1.53 & 3.09 & 88.66 \\
\hline 57671 & Yuanjiang & 28.85 & 112.37 & 37.00 & 112.55 & 653.90 & 1.52 & 4.07 & 83.97 \\
\hline 57682 & Pingjiang & 28.72 & 113.57 & 106.30 & 125.31 & 600.10 & 1.31 & 2.22 & 95.24 \\
\hline \multicolumn{10}{|c|}{ Lishui River } \\
\hline 57545 & Laifeng & 29.52 & 109.42 & 459.50 & 111.91 & 614.40 & 1.45 & 2.97 & 94.16 \\
\hline 57562 & Shimen & 29.58 & 111.37 & 116.90 & 113.01 & 595.10 & 1.58 & 2.84 & 97.28 \\
\hline \multicolumn{10}{|c|}{ Yuanjiang River } \\
\hline 57649 & Jishou & 28.32 & 109.73 & 208.40 & 116.15 & 558.00 & 1.44 & 2.21 & 94.16 \\
\hline 57655 & Yuanling & 28.47 & 110.40 & 151.60 & 118.13 & 503.70 & 1.46 & 2.02 & 99.03 \\
\hline 57662 & Changde & 29.05 & 111.68 & 35.00 & 122.38 & 2374.60 & 9.83 & 120.98 & 176.35 \\
\hline 57741 & Tongren & 27.72 & 109.18 & 279.70 & 105.37 & 415.80 & 1.12 & 0.87 & 83.70 \\
\hline 57745 & Zhijiang & 27.45 & 109.68 & 272.20 & 103.86 & 561.30 & 1.52 & 3.48 & 83.80 \\
\hline 57825 & Kaili & 26.60 & 107.98 & 720.30 & 100.62 & 497.60 & 1.23 & 1.31 & 84.68 \\
\hline 57832 & Sanhui & 26.97 & 108.67 & 626.90 & 91.98 & 367.30 & 1.16 & 1.10 & 69.87 \\
\hline 57845 & Tongdao & 26.17 & 109.78 & 397.50 & 120.54 & 510.00 & 1.39 & 1.92 & 94.65 \\
\hline \multicolumn{10}{|c|}{ Zishui River } \\
\hline 57669 & Anhua & 28.38 & 111.22 & 128.30 & 142.32 & 778.70 & 1.57 & 4.24 & 104.88 \\
\hline 57766 & Shaoyang & 27.23 & 111.47 & 248.60 & 110.00 & 471.90 & 1.17 & 1.59 & 81.00 \\
\hline 57853 & Wugang & 26.73 & 110.63 & 341.00 & 114.02 & 438.10 & 1.00 & 1.09 & 75.47 \\
\hline \multicolumn{10}{|c|}{ Xiangjiang River } \\
\hline 57774 & Shuangfeng & 27.45 & 112.17 & 100.00 & 112.64 & 484.20 & 1.24 & 1.78 & 83.14 \\
\hline 57776 & Nanyue & 27.30 & 112.70 & 1265.90 & 169.12 & 652.10 & 1.10 & 1.59 & 109.12 \\
\hline 57866 & Yongzhou & 26.23 & 111.62 & 172.60 & 117.52 & 497.60 & 1.16 & 1.68 & 85.92 \\
\hline 57872 & Hengyang & 26.90 & 112.60 & 104.90 & 111.35 & 615.80 & 1.27 & 3.09 & 79.62 \\
\hline 57965 & Daoxian & 25.53 & 111.60 & 192.20 & 126.17 & 568.20 & 1.11 & 1.28 & 96.72 \\
\hline
\end{tabular}

$$
Z_{M K}=\left\{\begin{array}{l}
\frac{S-1}{\sqrt{\operatorname{Var}(S)}} \rightarrow \text { ifS }>0 \\
0 \rightarrow \text { ifS }=0 \\
\frac{S+1}{\sqrt{\operatorname{Var}(S)}} \rightarrow \text { ifS }<0 \\
\text { if }<<0
\end{array}\right\}
$$

...where $X_{i}$ and $X_{j}$ represent sequential data values in the years $i$ and $j, n$ shows the length of series, $t_{p}$ is a number of bonds for the $\mathrm{p}^{\text {th }}$ value, and $\mathrm{q}$ shows the number of tied values. Positive values of $Z_{M K}$ and negative $Z_{M K}$ values postulate increasing and decreasing trends in the time series. The null hypothesis $\left(\mathrm{H}_{\mathrm{o}}\right)$ is rejected when $Z>Z_{1-\alpha / 2}$, and a significant trend exists in the time series. $Z_{1-\alpha / 2}$ is the acute value of $Z$ from the standard normal table, for 5\% significant level the value of $Z_{1-\alpha / 2}$ is 1.96 .

\section{Spearman's rho Test}

This test is also a rank-based statistical nonparametric technique applied for trend analysis and used for comparison purposes with the Mann-Kendall test [50]. In Spearman's rho test, which undertakes that time series data are autonomous or independent and distributed identically, the null hypothesis $\left(\mathrm{H}_{0}\right)$ yet again specifies no trend over time; the alternative hypothesis $\left(\mathrm{H}_{1}\right)$ is that a trend should be present and that data rise or fall with mathematical equations for calculating 
Table 3. MK and SR tests results for precipitation in monthly time series.

\begin{tabular}{|c|c|c|c|c|c|c|c|c|c|c|c|c|c|}
\hline Stations & TEST & JAN & FEB & MAR & APR & MAY & JUN & JUL & AUG & SEP & OCT & $\mathrm{NOV}$ & DEC \\
\hline \multirow{2}{*}{ Sangzhi } & MK & 1.98 & 1.00 & -0.21 & -1.03 & -0.67 & 0.40 & 1.02 & -0.80 & -0.70 & -0.29 & 0.40 & -1.29 \\
\hline & $\mathrm{Tt}$ & $2.08^{*}$ & 1.02 & -0.17 & -1.05 & -0.70 & 0.27 & 0.93 & -0.81 & -0.65 & -0.39 & 0.39 & -1.27 \\
\hline \multirow{2}{*}{ Nanxian } & MK & $2.37^{*}$ & 0.44 & -0.39 & 0.23 & -0.62 & -0.73 & $2.31^{*}$ & 0.20 & -0.66 & 0.17 & 0.17 & -0.99 \\
\hline & $\mathrm{Tt}$ & $2.53 *$ & 0.48 & -0.48 & 0.27 & -0.51 & -0.64 & $2.55^{*}$ & 0.21 & -0.69 & 0.03 & 0.26 & -1.17 \\
\hline \multirow{2}{*}{ Yueyang } & MK & $2.15^{*}$ & 1.05 & -0.96 & -0.15 & -0.02 & -0.81 & $2.63 *$ & 0.84 & 0.48 & -0.29 & 0.71 & -0.66 \\
\hline & $\mathrm{Tt}$ & $2.49 *$ & 1.17 & -1.10 & -0.14 & 0.09 & -0.71 & $2.89 *$ & 0.93 & 0.47 & -0.35 & 0.65 & -0.70 \\
\hline \multirow{2}{*}{ Yuanjiang } & MK & 1.91 & -0.01 & -0.88 & -0.89 & -0.58 & -0.81 & 1.70 & -0.12 & -0.59 & -0.12 & 0.84 & -0.67 \\
\hline & $\mathrm{Tt}$ & 1.93 & 0.00 & -0.90 & -0.83 & -0.57 & -0.70 & 1.75 & -0.24 & -0.46 & -0.29 & 0.83 & -0.83 \\
\hline \multirow{2}{*}{ Pingjiang } & MK & 1.91 & -0.31 & -1.03 & -1.40 & -1.16 & 0.81 & 1.08 & 1.41 & 0.17 & -0.79 & 0.54 & -0.13 \\
\hline & $\mathrm{Tt}$ & 1.88 & -0.25 & -0.96 & -1.48 & -1.15 & 0.82 & 0.88 & 1.54 & -0.08 & -0.70 & 0.53 & -0.11 \\
\hline \multirow{2}{*}{ Laifeng } & MK & 1.02 & 1.07 & -0.92 & -0.97 & -1.05 & -0.31 & 0.29 & -0.42 & -1.74 & -0.81 & -0.66 & -1.37 \\
\hline & $\mathrm{Tt}$ & 1.08 & 1.09 & -0.84 & -0.84 & -1.02 & -0.40 & 0.29 & -0.45 & -1.78 & -0.70 & -0.66 & -1.44 \\
\hline \multirow{2}{*}{ Shimen } & MK & 1.94 & 0.62 & -0.47 & -0.86 & -0.53 & 0.13 & 1.44 & -0.83 & -1.25 & 0.00 & 0.01 & -0.92 \\
\hline & $\mathrm{Tt}$ & 1.87 & 0.43 & -0.09 & -1.39 & -0.67 & 0.00 & 1.55 & -1.05 & -1.30 & -0.02 & -0.02 & -0.88 \\
\hline \multirow{2}{*}{ Jishou } & MK & 1.91 & -0.29 & -1.14 & $-2.14^{*}$ & -1.14 & 1.35 & 1.21 & -0.73 & -1.76 & -0.99 & -0.19 & -1.08 \\
\hline & $\mathrm{Tt}$ & $2.23 *$ & -0.71 & -1.01 & $-2.23 *$ & -0.87 & 1.07 & 1.23 & -0.91 & -1.80 & -1.15 & -0.36 & -1.01 \\
\hline \multirow{2}{*}{ Yuanling } & MK & 1.16 & -0.13 & -0.64 & -1.65 & -0.80 & 0.07 & 0.83 & -0.23 & -0.42 & 0.25 & 0.45 & -0.97 \\
\hline & $\mathrm{Tt}$ & 1.22 & -0.20 & -0.75 & -1.70 & -0.67 & 0.12 & 0.74 & -0.30 & -0.46 & 0.27 & 0.42 & -0.84 \\
\hline \multirow{2}{*}{ Changde } & MK & 1.94 & 0.66 & -0.77 & -0.28 & -0.20 & 0.24 & $2.47 *$ & -0.84 & -0.61 & -0.35 & 0.70 & -1.03 \\
\hline & $\mathrm{Tt}$ & $2.30^{*}$ & 0.73 & -0.92 & -0.35 & -0.11 & 0.36 & $2.63^{*}$ & -0.81 & -0.63 & -0.60 & 0.68 & -1.26 \\
\hline \multirow{2}{*}{ Tongren } & MK & 1.21 & 0.90 & 0.07 & $-2.94 *$ & -0.75 & 0.96 & 1.10 & -0.47 & -0.37 & $-2.34 *$ & -1.26 & -1.19 \\
\hline & $\mathrm{Tt}$ & $2.00 *$ & 0.42 & 0.83 & $-2.52 *$ & -1.72 & 1.32 & 0.97 & -0.38 & -0.31 & -1.82 & -1.17 & -1.08 \\
\hline \multirow{2}{*}{ Zhijiang } & MK & 1.85 & 0.54 & 1.11 & $-2.42 *$ & -1.60 & 1.11 & 1.03 & -0.47 & -0.40 & -1.71 & -1.35 & -1.02 \\
\hline & $\mathrm{Tt}$ & 1.80 & 0.25 & 0.17 & $-2.16^{*}$ & -1.72 & 0.56 & 1.00 & -0.74 & -1.92 & -1.21 & -0.61 & -0.79 \\
\hline \multirow{2}{*}{ Kaili } & MK & $2.29 *$ & 0.50 & 1.21 & -1.31 & -1.03 & 0.23 & 0.56 & -1.38 & -0.61 & -1.03 & -1.80 & -0.51 \\
\hline & $\mathrm{Tt}$ & $2.27^{*}$ & 0.48 & 1.30 & -1.50 & -1.09 & 0.29 & 0.43 & -1.39 & -0.69 & -0.99 & -1.90 & -0.72 \\
\hline \multirow{2}{*}{ Sanhui } & MK & 0.97 & 0.31 & 1.85 & -1.59 & -1.78 & 0.01 & 0.39 & $-2.11 *$ & 0.43 & -1.94 & -1.14 & -1.10 \\
\hline & $\mathrm{Tt}$ & 1.03 & 0.30 & 1.83 & -1.67 & -1.76 & -0.02 & 0.47 & -1.98 & 0.43 & -1.87 & -1.21 & -1.06 \\
\hline \multirow{2}{*}{ Tongdao } & MK & $2.56^{*}$ & 1.05 & 1.79 & $-2.63 *$ & 0.44 & 1.41 & 0.34 & -1.32 & -0.34 & -0.84 & -1.31 & -0.83 \\
\hline & $\mathrm{Tt}$ & $2.61 *$ & 1.18 & 1.74 & $-2.50 *$ & 0.43 & 1.41 & 0.28 & -1.18 & -0.17 & -0.84 & -1.21 & -0.81 \\
\hline \multirow{2}{*}{ Anhua } & MK & 1.77 & -0.39 & -0.28 & -0.84 & -0.43 & 1.10 & 1.78 & -1.30 & -0.70 & -0.84 & -0.02 & -0.84 \\
\hline & $\mathrm{Tt}$ & 1.83 & -0.31 & -0.43 & -0.76 & -0.28 & 1.08 & 1.86 & -1.29 & -0.76 & -0.94 & -0.12 & -0.53 \\
\hline \multirow{2}{*}{ Shaoyang } & MK & $2.18^{*}$ & -0.10 & 0.18 & $-3.24 *$ & -0.51 & 0.06 & 0.47 & -0.02 & 0.04 & -1.13 & -1.03 & -0.28 \\
\hline & $\mathrm{Tt}$ & $2.17 *$ & -0.09 & 0.13 & $-3.30 *$ & -0.64 & 0.08 & 0.36 & -0.09 & 0.21 & -1.09 & -0.89 & -0.25 \\
\hline \multirow{2}{*}{ Wugang } & MK & $2.03 *$ & 0.62 & 1.19 & $-2.55^{*}$ & -0.62 & -0.37 & 0.26 & -0.54 & -0.17 & -1.60 & -0.96 & -0.61 \\
\hline & $\mathrm{Tt}$ & $2.01 *$ & 0.49 & 1.13 & $-2.50 *$ & -0.68 & -0.21 & 0.30 & -0.62 & -0.16 & -1.51 & -0.92 & -0.64 \\
\hline \multirow{2}{*}{ Shuangfeng } & MK & $2.38 *$ & -0.07 & 0.32 & -1.92 & 0.21 & 0.75 & 1.11 & -0.18 & 0.79 & -1.05 & -0.61 & 0.02 \\
\hline & $\mathrm{Tt}$ & $2.47^{*}$ & -0.14 & 0.12 & -1.93 & 0.05 & 0.83 & 1.08 & -0.24 & 0.88 & -1.18 & -0.68 & 0.03 \\
\hline \multirow{2}{*}{ Nanyue } & MK & 0.75 & -0.88 & 0.62 & -1.93 & -0.39 & 1.18 & 0.66 & 0.88 & 1.13 & $-2.47 *$ & -1.56 & -0.77 \\
\hline & $\mathrm{Tt}$ & 0.91 & -0.83 & 0.65 & -1.77 & -0.59 & 1.22 & 0.50 & 0.83 & 1.17 & $-2.59 *$ & -1.59 & -0.77 \\
\hline
\end{tabular}


Table 3. Continued.

\begin{tabular}{|c|c|c|c|c|c|c|c|c|c|c|c|c|c|}
\hline \multirow{2}{*}{ Yongzhou } & $\mathrm{MK}$ & 1.80 & 0.36 & 1.08 & $-2.83 *$ & -0.04 & 0.92 & 0.84 & -0.29 & 0.06 & -1.68 & -0.70 & -0.72 \\
\cline { 2 - 13 } & $\mathrm{Tt}$ & 1.90 & 0.43 & 1.08 & $-2.76^{*}$ & 0.04 & 0.91 & 0.77 & -0.33 & -0.02 & -1.76 & -0.65 & -0.65 \\
\hline \multirow{2}{*}{ Hengyang } & $\mathrm{MK}$ & $2.23 *$ & 0.02 & 0.99 & -1.77 & 0.18 & 0.00 & 0.91 & -0.01 & 1.08 & -1.68 & -0.16 & -0.89 \\
\cline { 2 - 14 } & $\mathrm{Tt}$ & $2.38^{*}$ & 0.16 & 1.09 & -1.66 & 0.14 & 0.12 & 0.80 & 0.00 & 1.10 & -1.88 & -0.15 & -0.70 \\
\hline \multirow{2}{*}{ Daoxian } & $\mathrm{MK}$ & 1.60 & 0.28 & 0.12 & $-2.17 *$ & 0.48 & 0.53 & 1.08 & -0.88 & $-2.00 *$ & -1.13 & -0.55 & -0.85 \\
\cline { 2 - 13 } & $\mathrm{Tt}$ & 1.80 & 0.25 & 0.17 & $-2.16^{*}$ & 0.65 & 0.56 & 1.00 & -0.74 & -1.92 & -1.21 & -0.61 & -0.80 \\
\hline
\end{tabular}

$*$ Significant trend at $5 \%$ significance level of two-tailed test
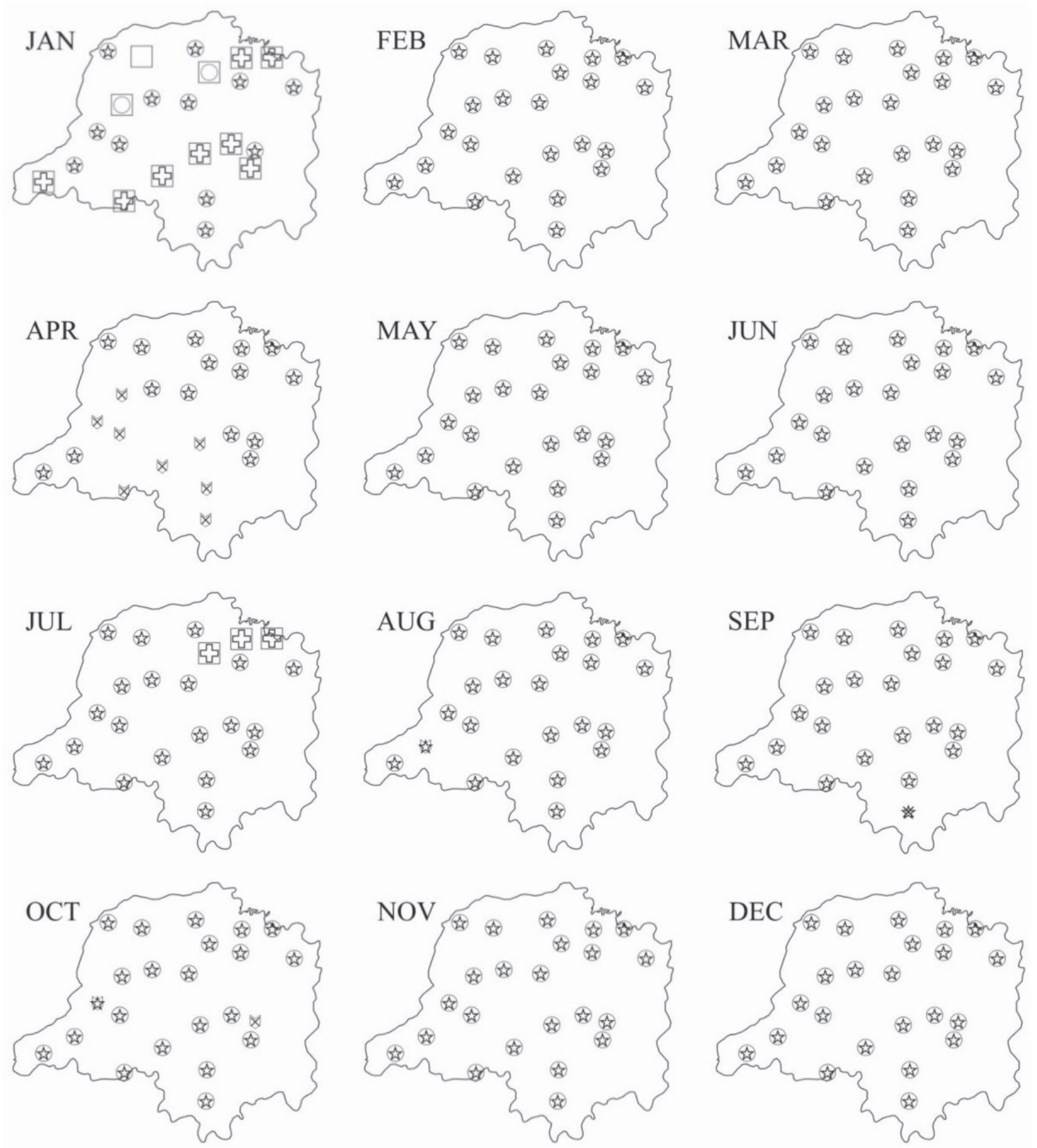

Fig. 2. Locations of stations with positive, negative, and no trends at 5\% significance level for the monthly precipitation time series. 
Spearman's rho statistical $R_{\mathrm{sp}}$ and standardized statistics $Z_{\mathrm{sp}}$ are given in the following equations:

$$
\begin{array}{r}
R_{s p}=1-\frac{6 \sum_{i=1}^{n}\left(D_{i}-i\right)^{2}}{n\left(n^{2}-1\right)} \\
Z_{s p}=R_{s p} \sqrt{\frac{n-2}{1-R_{s p}^{2}}}
\end{array}
$$

...where $D_{i}$ represents the rank of $i^{\text {th }}$ data observation, $i$ represents sequential order number, $n$ represents total length of the time series data, and $Z_{\mathrm{sp}}$ is Student's $t$-distribution with $(n-2)$ degree of freedom. Positive values of $Z_{\mathrm{sp}}$ and negative $Z_{\mathrm{sp}}$ values specify increasing and decreasing trends in the time series. The critical value of $t$ at a 0.05 significance level of Student's $t$-distribution table is defined as $(n-2,1-\alpha / 2)$. Null hypotheses $\left(\mathrm{H}_{0}\right)$ is rejected if $|Z \mathrm{sp}|>(n-2,1-\alpha / 2)$, and a significant trend occurs in time series.
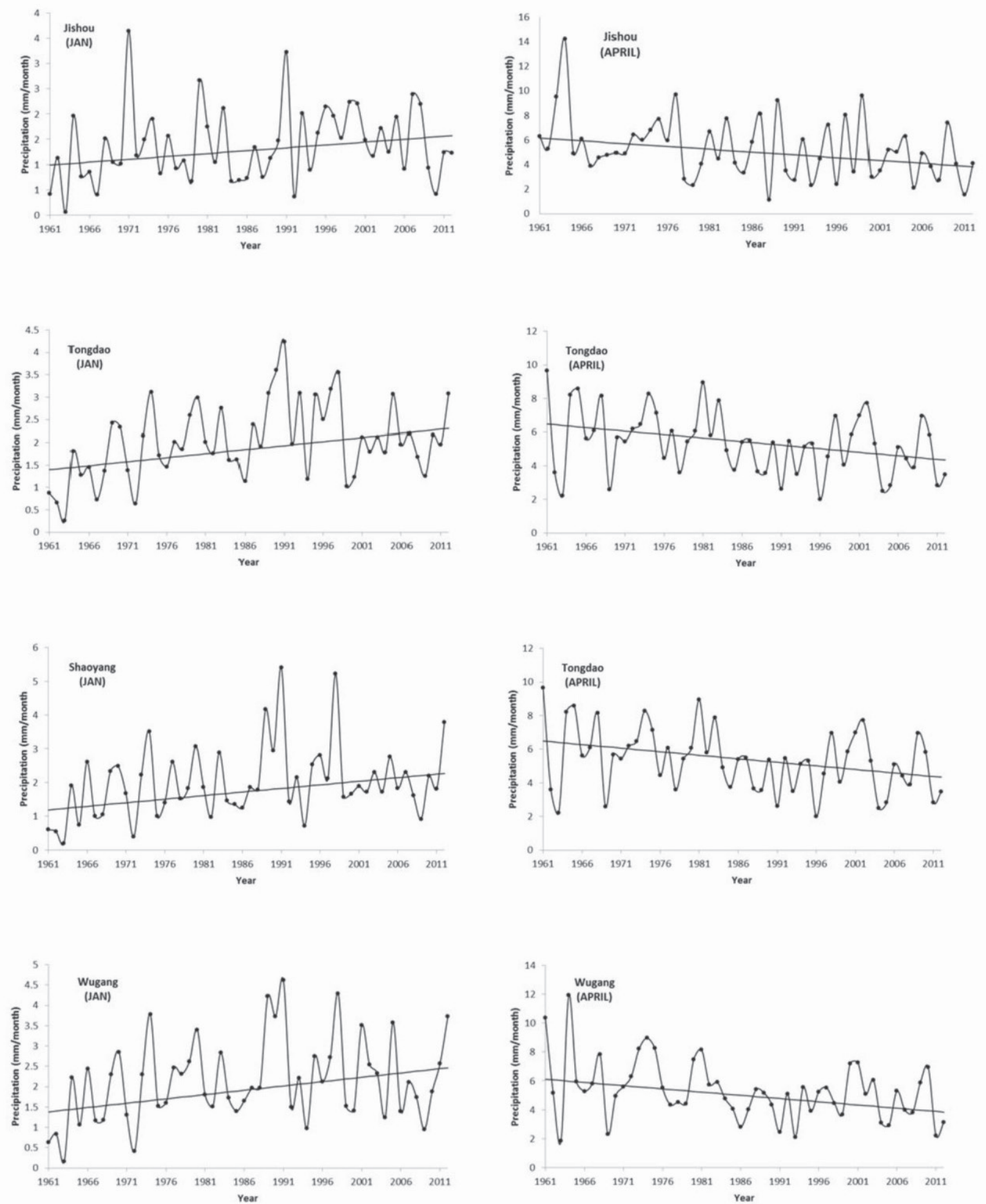

Fig. 3. Fluctuations in monthly precipitation time series in stations with the most significant trends during 1961-2012. 
Table 4. MK and SR tests results for precipitation annual time in seasonal and series.

\begin{tabular}{|c|c|c|c|c|c|c|c|}
\hline River Name & Station Name & TEST & Annual & Winter & Spring & Summer & Autumn \\
\hline \multirow{14}{*}{ Lake Region } & \multirow{2}{*}{ Sangzhi } & MK & 0.04 & 1.71 & -1.07 & 0.40 & -0.72 \\
\hline & & $\mathrm{Tt}$ & 0.11 & 0.32 & -0.94 & 0.62 & -0.77 \\
\hline & \multirow{2}{*}{ Nanxian } & MK & 0.31 & 1.32 & -0.65 & 0.81 & -0.56 \\
\hline & & $\mathrm{Tt}$ & 0.31 & 1.39 & -0.67 & 0.84 & -0.64 \\
\hline & \multirow{2}{*}{ Yueyang } & MK & 1.03 & 1.91 & -0.71 & 0.97 & 0.32 \\
\hline & & $\mathrm{Tt}$ & 1.03 & $2.08^{*}$ & -0.68 & 0.98 & 0.30 \\
\hline & \multirow{2}{*}{ Yuanjiang } & MK & -0.09 & 1.22 & -1.40 & 0.64 & 0.21 \\
\hline & & $\mathrm{Tt}$ & -0.02 & 1.24 & -1.34 & 0.58 & 0.20 \\
\hline & \multirow{2}{*}{ Pingjiang } & MK & -0.09 & 1.21 & -1.87 & 1.93 & -0.21 \\
\hline & & $\mathrm{Tt}$ & -0.16 & 1.28 & $-2.02 *$ & 1.95 & -0.14 \\
\hline & \multirow{2}{*}{ Laifeng } & MK & -1.32 & 1.00 & -1.38 & -0.07 & $-2.22 *$ \\
\hline & & $\mathrm{Tt}$ & -1.28 & 0.90 & -1.49 & -0.19 & $-2.19^{*}$ \\
\hline & \multirow{2}{*}{ Shimen } & MK & -0.29 & 0.94 & -1.29 & 0.80 & -1.35 \\
\hline & & $\mathrm{Tt}$ & -0.26 & 1.05 & -1.14 & 0.82 & -1.40 \\
\hline \multirow{16}{*}{ Yuanjiang River } & \multirow{2}{*}{ Jishou } & MK & -1.03 & 0.68 & $-2.30^{*}$ & 0.83 & -1.73 \\
\hline & & $\mathrm{Tt}$ & -1.05 & 0.58 & -2.37 & 0.94 & -1.68 \\
\hline & \multirow{2}{*}{ Yuanling } & MK & -0.92 & 0.19 & -1.81 & 0.77 & -0.02 \\
\hline & & $\mathrm{Tt}$ & -0.84 & 0.04 & -1.72 & 0.57 & -0.05 \\
\hline & \multirow{2}{*}{ Changde } & MK & 0.67 & 1.39 & -0.65 & 0.78 & -0.24 \\
\hline & & $\mathrm{Tt}$ & 0.47 & 1.36 & -0.65 & 0.84 & -0.34 \\
\hline & \multirow{2}{*}{ Tongren } & MK & -1.08 & 0.89 & $-2.53^{*}$ & 1.00 & $-2.19^{*}$ \\
\hline & & $\mathrm{Tt}$ & -1.19 & 1.18 & $-2.63^{*}$ & 1.04 & $-2.33^{*}$ \\
\hline & \multirow{2}{*}{ Zhijiang } & MK & -1.41 & 0.90 & $-2.51^{*}$ & 0.99 & -1.13 \\
\hline & & $\mathrm{Tt}$ & -1.52 & 0.99 & $-2.79^{*}$ & 0.87 & -1.34 \\
\hline & \multirow{2}{*}{ Kaili } & MK & -1.65 & 1.03 & -1.40 & -0.29 & -1.88 \\
\hline & & $\mathrm{Tt}$ & -1.69 & 1.44 & -1.56 & -0.22 & -1.94 \\
\hline & \multirow{2}{*}{ Sanhui } & MK & -1.74 & 0.43 & -1.60 & -0.36 & -1.58 \\
\hline & & $\mathrm{Tt}$ & -0.09 & 1.94 & -0.76 & 0.68 & -0.90 \\
\hline & \multirow{2}{*}{ Tongdao } & MK & 0.00 & 1.78 & -0.81 & 0.69 & -0.74 \\
\hline & & $\mathrm{Tt}$ & -0.09 & 1.94 & -0.76 & 0.68 & -0.90 \\
\hline \multirow{6}{*}{ Zishui River } & \multirow{2}{*}{ Anhua } & MK & 0.04 & 1.03 & -0.61 & 0.65 & -0.95 \\
\hline & & $\mathrm{Tt}$ & 0.06 & 0.95 & -0.57 & 0.70 & -1.11 \\
\hline & \multirow{2}{*}{ Shaoyang } & MK & -0.51 & 1.60 & -1.84 & 0.49 & -1.29 \\
\hline & & $\mathrm{Tt}$ & -0.57 & 1.69 & $-2.00 *$ & 0.47 & -1.18 \\
\hline & \multirow{2}{*}{ Wugang } & MK & -1.29 & 1.23 & -1.78 & -0.06 & -1.51 \\
\hline & & $\mathrm{Tt}$ & -1.29 & 1.75 & -1.76 & -0.17 & -1.54 \\
\hline \multirow{4}{*}{ Xiangjiang River } & \multirow{2}{*}{ Shuangfeng } & MK & 1.03 & 1.62 & -0.59 & 1.35 & -0.86 \\
\hline & & $\mathrm{Tt}$ & 0.92 & 1.73 & -0.73 & 1.39 & -0.89 \\
\hline & \multirow{2}{*}{ Nanyue } & MK & 0.15 & -0.07 & -0.05 & 1.37 & -1.45 \\
\hline & & $\mathrm{Tt}$ & -0.02 & -0.01 & -0.24 & 1.44 & -1.49 \\
\hline
\end{tabular}


Table 4. Continued.

\begin{tabular}{|c|c|c|c|c|c|c|c|}
\hline \multirow{3}{*}{ Xiangjiang River } & \multirow{3}{*}{ Yongzhou } & $\mathrm{MK}$ & 0.02 & 0.92 & -0.67 & 1.11 & -1.14 \\
\cline { 3 - 9 } & \multirow{3}{*}{ Hengyang } & $\mathrm{Tt}$ & -0.04 & 1.12 & -0.71 & 1.22 & -1.30 \\
\cline { 3 - 9 } & & $\mathrm{MK}$ & -0.17 & 1.29 & -0.02 & 0.12 & -1.05 \\
\cline { 3 - 9 } & \multirow{3}{*}{ Daoxian } & $\mathrm{Tt}$ & -0.29 & 1.43 & -0.16 & 0.06 & -0.94 \\
\cline { 3 - 9 } & & $\mathrm{MK}$ & -0.62 & 0.89 & -0.86 & 0.65 & -1.84 \\
\cline { 3 - 9 } & & $\mathrm{Tt}$ & -0.45 & 0.82 & -0.69 & 0.58 & $-2.00 *$ \\
\hline
\end{tabular}

*Significant trend at 5\% significance level of two-tailed test

\section{Sen's Slope Estimator}

This nonparametric technique was applied to assess the scale of trends in the time series data:

$$
T_{i}=1-\frac{x_{j}-x_{k}}{j-k}
$$

...where $x_{j}$ and $x_{k}$ represent data values at time $j$ and $k$, respectively. Consider positive values of $Q_{i}$ and negative $Q_{i}$ values specify increasing and decreasing trends in the time series Eq. 6.

$$
Q_{i}=\left\{\begin{array}{c}
T_{(N+1) / 2} \\
\frac{1}{2}\left(T_{N / 2}+T_{(N+2) / 2}\right)
\end{array}\right\}
$$

\section{Results and Discussions}

\section{Monthly Analysis}

The MK and Spearman's rho (Tt) tests were used on a monthly scale to identify trends in precipitation series at different stations. Table 3 shows the results and illustrates that the results of both tests were similar to one another. The magnitude of statistically significant trends on a monthly scale was detected using Sen's slope estimator. At different stations, monthly trend tests showed a mix of positive and negative trends.

At Sangzhi, Kaili, Shuangfeng, and Hengyang stations statistically significant positive trends were found in January, and no significant trends were detected for other months. Significant positive trends were detected at Nanxian, Yueyang, and Changde stations in January and July. Jishou, Tongdao, Shaoyang, and Wuganag stations exhibited statistically significant positive and negative trends in January and April, respectively. In April, Zhijiang station showed a significant negative trend. Tongren Station exhibited a significant positive trend in January but had a significant negative trend in April and October. Like this station, Nanyue is the only one that shwoed a significant negative trend in the month of October. Stations that showed statistically significant negative trends in August include Sanhui and Yongzhou. Significant negative trends were detected at Daoxian Station in April and November. All remaining stations had not shown any statistically significant trends in any case in any month. Fig. 2 shows the spatial

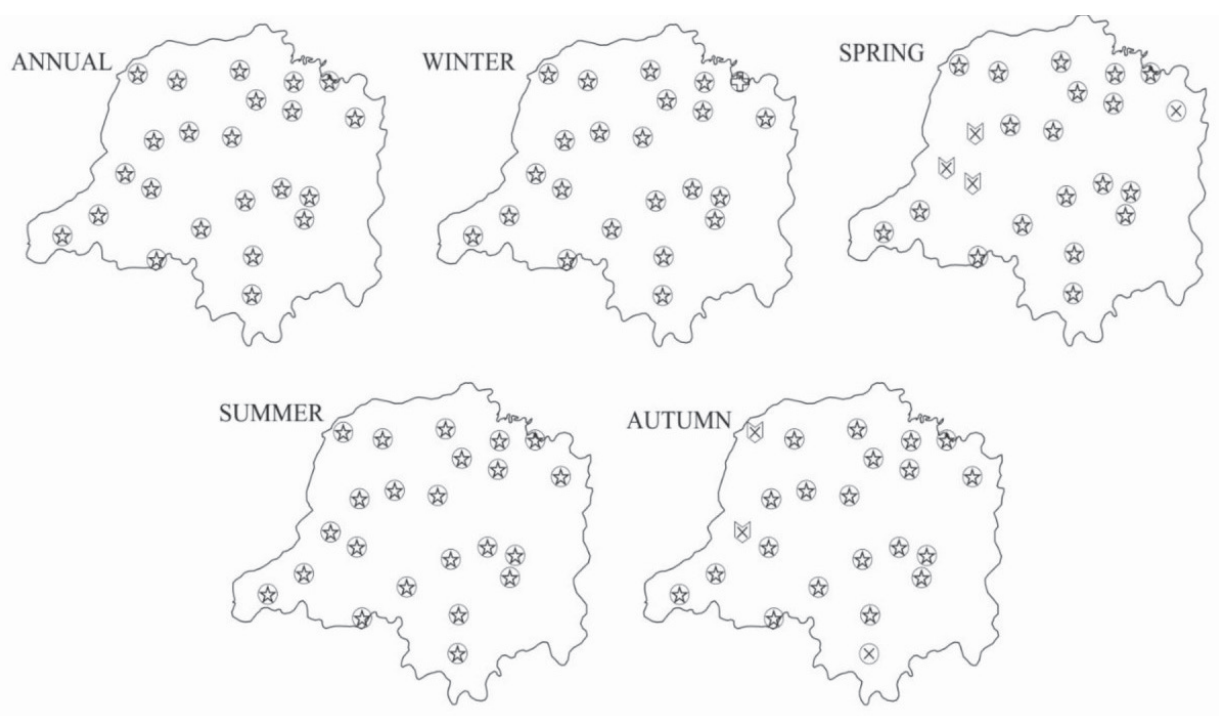

Fig. 4. Locations of stations with positive, negative, and no trends at $5 \%$ significance level for seasonal and annual precipitation times. 
variation in the monthly precipitation time series in the Dongting region from 1961 to 2012. The results show that trends at Nanxian and Daoxian stations were rapid as compared to other stations. Nanxian station had the maximum positive increase in monthly precipitation (1.86 $\mathrm{mm} / \mathrm{month})$ and maximum negative decline (0.032 $\mathrm{mm} / \mathrm{month})$ during the months of July and September. Jishou, Tongdao, Shaoyang, and Wugang stations showed a sharp change from significantly positive to negative trends in January and April, respectively. Fig. 3 shows the trends in maximum monthly precipitation variation at the different stations.

\section{Seasonal and Annual Analysis}

The MK and Spearman's rho tests were also used to identify trends in seasonal and annual precipitation from 1961 to 2012 (Table 4). Similar to the monthly analysis, results from both statistical tests, MK and SR, were consistent with one another. A mix of negative and positive trends was seen at different stations. Many stations that had shown a great deal of significance in monthly precipitation time series did not exhibit significant seasonal and annual trends.

Most importantly for the annual precipitation series, no significant trends were detected at any station. The statistically significant positive trend was detected at Yueyang Station in the winter. At Pinjiang, Jishou, Zhijiang, and Shaoyang stations, there were statistically significant negative trends in the spring. At Tongren, significant negative trends were detected in both spring and autumn. At Daoxian, a significant negative trend was detected in the autumn.

All other stations showed no significant trends in seasonal as well as annual precipitation time series. Fig. 4 presents the spatial distribution of seasonal
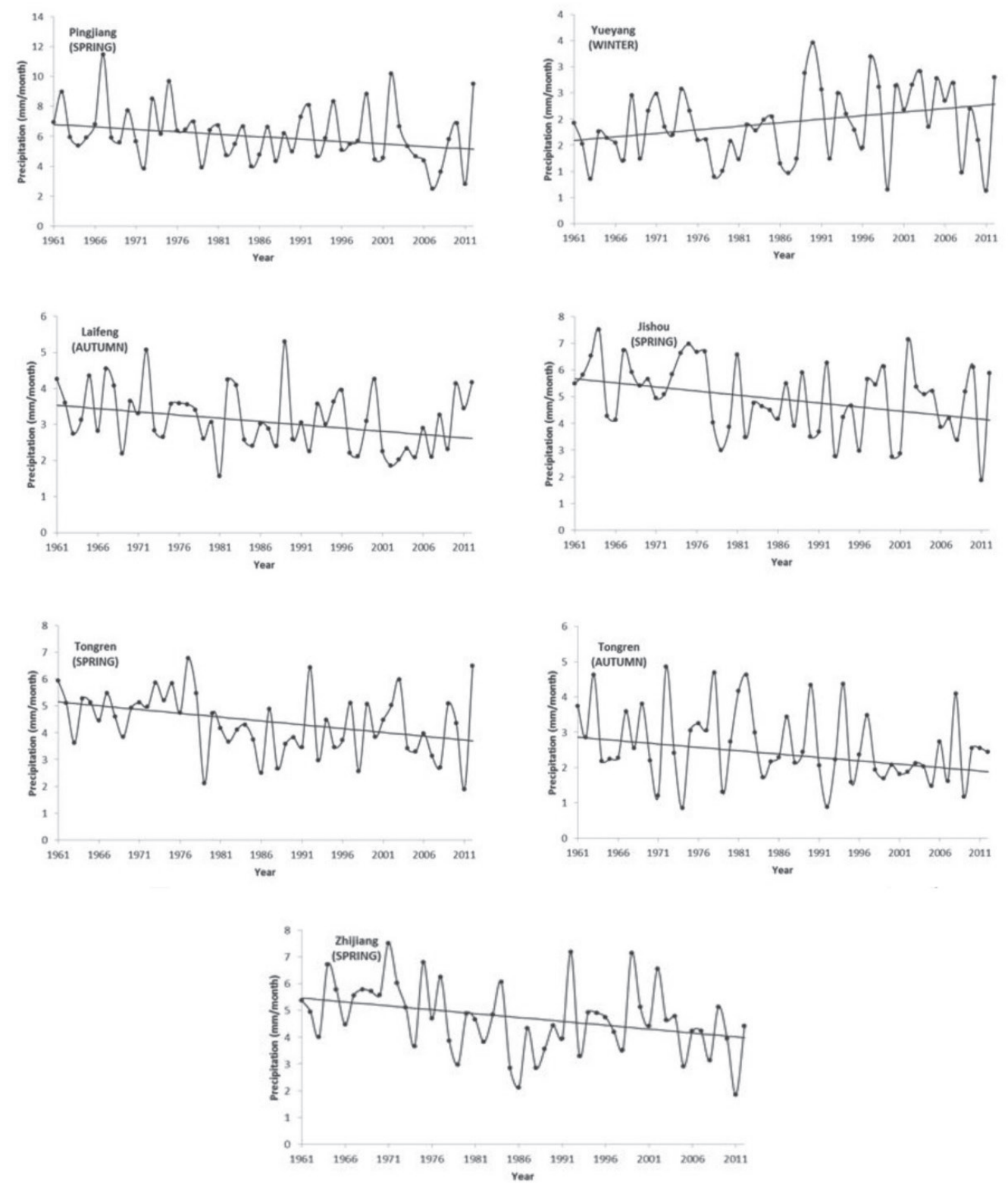

Fig. 5. Fluctuations in seasonal and annual precipitation time series in stations with the most significant trends during 1961-2012. 
Table 5. MK and SR tests results for monthly precipitation time series in the entire Dongting basin and sub-basin.

\begin{tabular}{|c|c|c|c|c|c|c|c|c|c|c|c|c|c|}
\hline River Name & TEST & JAN & FEB & MAR & APR & MAY & JUN & JUL & AUG & SEP & OCT & NOV & DEC \\
\hline \multirow{2}{*}{ Lake Region } & MK & $2.22 *$ & 0.54 & -0.73 & -0.92 & -0.80 & -0.28 & 1.96 & 0.53 & -0.40 & -0.26 & 0.58 & -0.77 \\
\hline & $\mathrm{Tt}$ & $2.29^{*}$ & 0.57 & -0.89 & -0.92 & -0.81 & -0.25 & $2.12 *$ & 0.53 & -0.42 & -0.29 & 0.53 & -0.89 \\
\hline \multirow{2}{*}{ Lishui River } & MK & 1.63 & 0.83 & -0.37 & -0.67 & -0.34 & 0.29 & 1.07 & -0.92 & -1.52 & -0.20 & -0.59 & -1.29 \\
\hline & $\mathrm{Tt}$ & 1.64 & 0.85 & -0.41 & -0.73 & -0.58 & 0.28 & 1.09 & -1.00 & -1.57 & -0.25 & -0.47 & -1.23 \\
\hline \multirow{2}{*}{$\begin{array}{l}\text { Yuanjiang } \\
\text { River }\end{array}$} & MK & $2.09^{*}$ & 0.58 & 0.36 & $-2.17^{*}$ & -1.21 & 0.59 & 1.48 & -1.54 & -0.95 & -1.82 & -1.11 & -1.33 \\
\hline & $\mathrm{Tt}$ & $2.13^{*}$ & 0.57 & 0.28 & $-2.36^{*}$ & -1.19 & 0.65 & 1.48 & -1.44 & -0.96 & -1.93 & -1.09 & -1.19 \\
\hline \multirow{2}{*}{ Zishui River } & MK & $2.08^{*}$ & 0.02 & 0.26 & 0.86 & -0.15 & 0.37 & 1.10 & -0.70 & -0.54 & -1.43 & -0.70 & -0.70 \\
\hline & $\mathrm{Tt}$ & $2.15^{*}$ & 0.04 & 0.18 & 0.97 & -0.59 & 0.49 & 1.10 & -0.80 & -0.47 & -1.43 & -0.65 & -0.58 \\
\hline \multirow{2}{*}{$\begin{array}{l}\text { Xiangjiang } \\
\text { River }\end{array}$} & MK & 1.92 & -0.17 & 0.53 & $-2.35^{*}$ & 0.23 & 1.18 & 1.21 & -0.18 & 0.43 & -1.92 & -0.59 & -1.33 \\
\hline & $\mathrm{Tt}$ & $2.06^{*}$ & -0.05 & 0.52 & $-2.30 *$ & 0.20 & 1.11 & 1.07 & -0.25 & 0.48 & $-2.01 *$ & -0.66 & -1.25 \\
\hline \multirow{2}{*}{$\begin{array}{l}\text { Dongting } \\
\text { Basin }\end{array}$} & MK & $2.11^{*}$ & 0.50 & -0.04 & -1.44 & -0.61 & 0.39 & $2.09^{*}$ & -0.67 & -1.07 & -1.40 & -0.28 & -1.00 \\
\hline & $\mathrm{Tt}$ & $2.46^{*}$ & 0.48 & -0.10 & -1.42 & -0.66 & 0.39 & $2.13^{*}$ & -0.68 & -1.09 & -1.55 & -0.35 & -1.03 \\
\hline
\end{tabular}

"Significant trend at 5\% significance level of two-tailed test.

precipitation trends. Fig. 5 shows the trend magnitude in seasonal precipitation variation at the different stations.

\section{Trend Analysis over the River Basin}

The effect of climate change on precipitation was also analyzed for the entire Dongting region basin by applying MK and Spearman's rho (Tt) tests on monthly, seasonal, and annual scales. As Fig. 1 showed, the entire Dongting River catchment (A) is divided into five subbasins: Lake Region (A1), Lishui River (A2), Yuanjiang
River (A3), Zishui River (A4), and Xianjiang River (A5). Table 5 presents trends in monthly precipitation across the sub-basins and for the entire basin.

Table 5 shows significant positive trends in the months of January and July for the Lake Region (A1). In the Lishui River (A2), statistically no-significant trends were identified. In the Yuanjiang River (A3), statistically significant positive and negative trends were recognized in January and April. The Zishui River (A4) showed statistically significant positive trends in January. The maximum numbers of significant cases (three) were observed in results from the Xianjiang River (A5), which

Table 6. MK and SR results for seasonal and annual precipitation time series in the entire Donting basin and sub-basin.

\begin{tabular}{|c|c|c|c|c|c|c|}
\hline River Name & TEST & ANNUAL & WINTER & SPRING & SUMMER & AUTUMN \\
\hline \multirow{3}{*}{ Lake Region } & $\mathrm{MK}$ & 0.20 & 0.75 & -1.67 & 1.07 & -0.13 \\
\cline { 2 - 7 } & $\mathrm{Tt}$ & 0.19 & 0.45 & -1.62 & 1.07 & -0.22 \\
\hline \multirow{3}{*}{ Lishui River } & $\mathrm{MK}$ & -0.80 & -1.21 & -1.62 & 0.47 & -1.85 \\
\cline { 2 - 7 } & $\mathrm{Tt}$ & -0.76 & -1.30 & -1.47 & 0.51 & -1.90 \\
\hline \multirow{3}{*}{ Zuanjiang River } & $\mathrm{MK}$ & -0.91 & -1.60 & $-2.34^{*}$ & 0.50 & -1.60 \\
\cline { 2 - 7 } & $\mathrm{Tt}$ & -0.93 & -1.65 & $-2.28^{*}$ & 0.46 & -1.63 \\
\hline \multirow{2}{*}{ Xiangjiang River River } & $\mathrm{MK}$ & 0.00 & -0.06 & -0.15 & 0.53 & -1.44 \\
\cline { 2 - 7 } & $\mathrm{Tt}$ & -0.02 & -0.07 & -0.38 & 0.48 & -1.46 \\
\hline \multirow{2}{*}{ Dongting Basin } & $\mathrm{MK}$ & 0.17 & 0.69 & -0.64 & 0.95 & -1.27 \\
\cline { 2 - 7 } & $\mathrm{Tt}$ & 0.14 & 0.74 & -0.71 & 1.00 & -1.23 \\
\hline
\end{tabular}

"Significant trend at $5 \%$ significance level of two-tailed test. 
showed a significant positive trend during January and significant negative trends were identified in April and October.

In the entire Dongting Basin (A), statistically significant positive trends were detected in January and July. As a simpler summary, significant positive trends in precipitation over time were seen in January and July; negative trends over time were seen in April and October in different Dongting sub-basins. To detect precipitation trends in each sub-basin and the entire Dongting basin, the Mann-Kendall and Spearman's rho tests were again applied to seasonal and annual precipitation data for the 52-year study period (1961-2012) at 23 meteorological stations.

As Table 6 shows, both test methods showed similar results. Except for Yuanjiang River (A3), the other entire Dongting River catchment (A), Lake Region (A1), Lishui River (A2), Zishui River (A4), and Xianjiang River (A5) showed statistically insignificant positive or negative trends. Only Yuanjiang River (A3) showed a significant
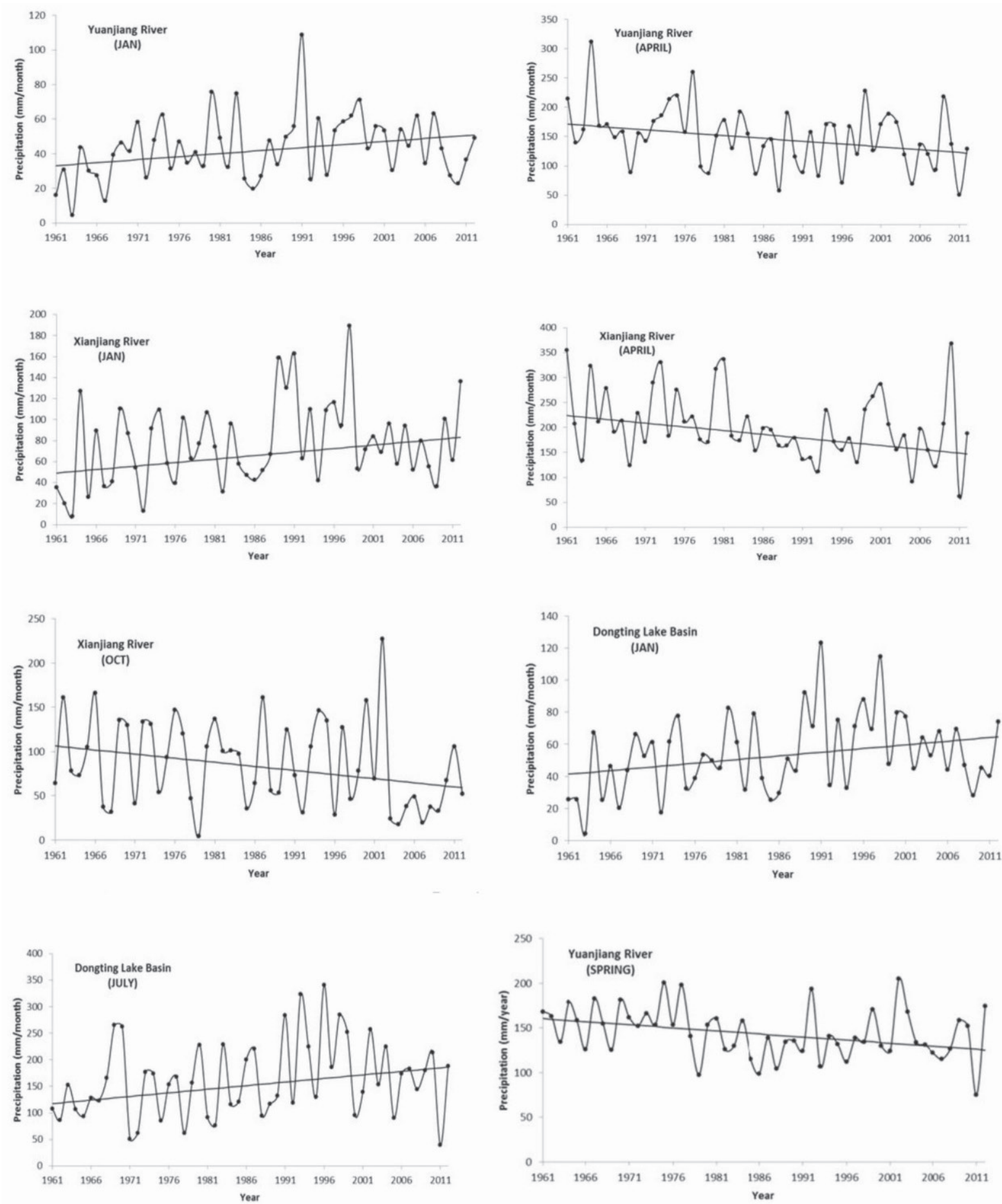

Fig. 6. Fluctuations in monthly and seasonal precipitation time series in basins and sub-basins with significant trends during 1961-2012. 
negative trend in the spring season. Fig. 6 shows the trends in maximum monthly precipitation variation in the whole Dongting basin and sub-basins.

\section{Comparing Results}

Results from both statistical tests, MK and Spearman's rho tests were in agreement with each other. The percentages of statistically significant trends cases to total verified cases for the MK and Spearman's rho tests were $8.20 \%$ and $8.30 \%$, respectively, showing agreement across methods [51].

\section{Discussion}

This study investigated variability in monthly, seasonal, and annual precipitation at 23 stations in the Dongting basin over a 52-year study period (1961-2012). Precipitation trends were also analyzed for each sub-basin and the entire Dongting Lake basin. The mean annual precipitation at different stations showed considerable variation.

1. The Nanxian, Yueyang, Changde, Kaili, Shuangfeng and Hengyang stations showed significant positive trends (increased precipitation over time) at 5\% significance level in the annual precipitation series.

2. Shaoyang station showed the maximum decreasing slope (indicating sharpest change over time) of $-0.06 \mathrm{~mm} /$ year among the selected stations in Dongting Lake Basin.

3. These variations in precipitation trends may lead China toward more water-related disasters such as droughts and floods in the near future. Basistha et al. [52] proposed possible causes of the changing precipitation trends, such as global climate shifts, dwindling global monsoon circulation, the decline in forest cover [53], use changes and practices (e.g., irrigated agriculture), and increasing aerosols from anthropogenic activities [54]. Findings were also consistent with the studies by Song et al. [55] and Tian et al. [56] in northwestern Zhejiang, where a mix positive and negative trends of precipitation for annual and seasonal data was found.

4. The results of the present study were found to be consistent as predicted in previous studies [57, 58], in which significant positive trends detected in precipitation time series during the 20th century. The mostly grid stations at high elevation exhibit higher temperature and precipitation trends and follow the same results found by Tang et al. [59].

5. These positive trends can affect the water resources of the area. Runoff in the study area did not significantly increase (increase insignificantly) due to positive trends in precipitation and temperature, and there was no shortage of water demands for agriculture, hydropower, and domestic use. Also, this increasing water flow can cause flooding in the area, as stated by Piao et al. [60] in the $20^{\text {th }}$ century.

\section{Acknowledgements}

This research has been supported by the Nonprofit Industry Financial Program of the Ministry of Water Resources of China (No. 201301066), the National Natural Science Foundation of China (Nos. 40701024, 41101511, and 51409152), and the Hubei Provincial Collaboration Innovation Center for Water Security.

\section{Conflict of Interest}

The authors declare no conflict of interest.

\section{References}

1. JIANG B, CHEN J, LUO Q, LAI J, XU H, WANG Y, YU K. Long-Term Changes in Water Quality and Eutrophication of China's Liujiang River. Pol. J. Environ. Stud. 25 (3), 1033, 2016.

2. MANN HB. Nonparametric tests against trend. Econom J Econom Soc. 245, 1945

3. SEN P.K. Estimates of the regression coefficient based on Kendall's tau. J Am Stat Assoc. 63, 1379, 1968.

4. YANG X.L., XU L.R., LIU K.K., LI C.H., HU J., XIA X.H. Trends in temperature and precipitation in the Zhangweinan River Basin during the last 53 Years. Procedia Environ Sci. 13, 1966, 2012.

5. YANG Z.F., YAN Y., LIU Q. The relationship of Streamflow-Precipitation-Temperature in the Yellow River Basin of China during 1961-2000. Procedia Environ Sci. 13, 2336, 2012.

6. TAO H., GEMMER M., BAI Y., SU B., MAO W. Trends of streamflow in the Tarim River Basin during the past 50 years: Human impact or climate change? J Hydrol. 400, 1, 2011.

7. YANING C., CHANGCHUN X., XINGMING H., WEIHONG L., YAPENG C., CHENGGANG Z., ZHAOXIA Y. Fifty-year climate change and its effect on annual runoff in the Tarim River Basin, China. Quat Int. 208, 53, 2009.

8. SHADMANI M., MAROFI S., ROKNIAN M. Trend analysis in reference evapotranspiration using MannKendall and Spearman's Rho tests in arid regions of Iran. Water Resour Manag. 26, 211, 2012.

9. CÁRDENAS O.L., CAMPOS M.N., PATRICIA N., SEVILLA M. Hydroclimatic Trends in Areas with High Agricultural Productivity in Northern Mexico. 24, 1165, 2015.

10. ZHAO S., FANG J. Impact of Impoldering and in Changes Central Yangtze Restoration on Dongting Lake. 2004.

11. DU Y., CAI S., ZHANG X., ZHAO Y. Interpretation of the environmental change of Dongting Lake, middle reach of Yangtze River, China, by $210 \mathrm{~Pb}$ measurement and satellite image analysis. Geomorphology. 41, 171, 2001.

12. CHEN J., WU X., FINLAYSON B.L., WEBBER M., WEI T., LI M., CHEN Z. Variability and trend in the hydrology of the Yangtze River, China: Annual precipitation and RUNOFF. J HYDROL. 513, 403, 2014.

13. XU J., YANG D., LIU Z., LEI Z. Analysis of SpatialTemporal Variability of Water Resources in Upstream of Yangtze River Using Distributed Hydrological Model. J China Hydrol. 27, 10, 2007. 
14. WANG Y., ZHOU L. Observed trends in extreme precipitation events in China during 1961-2001 and the associated changes in large-scale circulation. Geophys Res Lett. 32, 1, 2005.

15. XU C., FAN K., XIAO T. Runoff characteristics and variation tendency of Jinsha River Basin. Yangtze River. 7 (4), 2010.

16. XU Z.X., LI J.Y., LIU C.M. Long-term trend analysis for major climate variables in the Yellow River basin. Hydrol PROCESS. JOHN WILEY \& SONS, LTD. 21, 1935, 2007.

17. ALEKSANYAN A.S., KHUDAVERDYAN S.K., VASEASHTA A. Modeling River Ecosystem Vulnerability Assessments Due to Climate Change: Case Study of Armenia. 24, 871, 2015.

18. ADNAN M., NABI G., KANG S., ZHANG G., ADNAN R.M., ANJUM M.N., IQBAL M., ALI A.F. Snowmelt Runoff Modelling under Projected Climate Change Patterns in the Gilgit River Basin of Northern Pakistan. 26, 525, 2017.

19. YUNLING H., YIPING Z. Climate Change from 1960 to 2000 in the Lancang River Valley, China. Mt Res Dev. 25, 341, 2015.

20. GU Y., LIN J., WANG X.L., ZHANG X.J. Trend of annual runoff for major rivers in China under climate change. Procedia Eng. 28, 564, 2012.

21. FAN X., WANG M. Change trends of air temperature and precipitation over Shanxi province, China. Theor Appl Climatol. 103, 519, 2011.

22. JIANG Y., LUO Y., ZHAO Z., TAO S. Changes in wind speed over China during 1956-2004. Theor Appl Climatol. 99, 421, 2010.

23. MISHRA N., KUMAR M., KUMAR S. Trend Analysis of Precipitation by MK Test in Kumaon Region of Uttarakhand (1901-2010). Indian J Sci Technol. 9, 2016.

24. AHMAD I., TANG D., WANG T., WANG M., WAGAN B. Precipitation trends over time using Mann-Kendall and spearman's Rho tests in swat river basin, Pakistan. Adv Meteorol. 2015, 1, 2015.

25. PALIZDAN N., FALAMARZI Y., HUANG Y.F., LEE T.S., GHAZALI A.H. Regional precipitation trend analysis at the Langat River Basin, Selangor, Malaysia. Theor Appl Climatol. Springer Vienna; 117, 589, 2014.

26. PALIZDAN N., FALAMARZI Y., HUANG Y.F., LEE T.S. Precipitation trend analysis using discrete wavelet transform at the Langat River Basin, Selangor, Malaysia. Stoch Environ Res Risk Assess. Springer Berlin Heidelberg; 31, 8537, 2017.

27. RASHID M.M., BEECHAM S., CHOWDHURY R.K. Assessment of trends in point rainfall using Continuous Wavelet Transforms. Adv Water Resour. 82, 1, 2015.

28. IRANNEZHAD M., TORABI HAGHIGHI A., CHEN D., KLØVE B. Variability in dryness and wetness in central Finland and the role of teleconnection patterns. Theor Appl Climatol. 122, 471, 2015.

29. RIM C.-S. Homogeneity of Climate Aridity Index Trends Using Mann-Kendall Trend Test. J Korea Water Resour Assoc. 47, 643, 2014.

30. SINGH P., KUMAR V., THOMAS T., ARORA M. Changes in rainfall and relative humidity in river basins in northwest and central India. Hydrol Process. 22, 2982, 2008.

31. SHIFTEH SOME'E B., EZANI A., TABARI H. Spatiotemporal trends and change point of precipitation in Iran. Atmos Res. 113, 1, 2012.

32. PARTAL T., KAHYA E. Trend analysis in Turkish precipitation data. Hydrol Process. 20, 2011, 2006.
33. DJAMAN K., BALDE A.B., RUDNICK D.R., NDIAYE O., IRMAK S. Long-term trend analysis in climate variables and agricultural adaptation strategies to climate change in the Senegal River Basin. Int J Climatol. 37, 2873, 2017.

34. CÁRDENAS O.L., CAMPOS M.N., SEVILLA P.M., GUERRERO R.R., OCAMPO H.A.G., MORENO M.N.H. Estimating Trends and Return Periods of Daily Extreme Precipitation Associated with Tropical Cyclones in the Core North American Monsoon. Pol. J. Environ. Stud. 25, 2283, 2016.

35. CÁRDENAS O.L., GUEVARA H.J.P, MONTOYA J.M., CAMPOS M.N., OCAMPO H.A.G. Seasonal trend indicators and return periods of meteorological drought in the northern states of Mexico. 26, 1471, 2017.

36. FATHIAN F., DEHGHAN Z., BAZRKAR M.H., ESLAMIAN S. Trends in hydrologic and climatic variables affected by four variations of Mann-Kendall approach in Urmia Lake basin, Iran. Hydrol Sci J. 6667, 2014.

37. DINPASHOH Y., MIRABBASI R., JHAJHARIA D., ABIANEH H.Z., MOSTAFAEIPOUR A. Effect of ShortTerm and Long-Term Persistence on Identification of Temporal Trends. J Hydrol Eng. 19, 617, 2014.

38. ZHAO G., HÖRMANN G., FOHRER N., ZHANG Z., ZHAI J. Streamflow trends and climate variability impacts in Poyang Lake basin, China. Water Resour Manag. 24, 689, 2010.

39. HASSANZADEH E., ZARGHAMI M., HASSANZADEH Y. Determining the Main Factors in Declining the Urmia Lake Level by Using System Dynamics Modeling. Water Resour Manag. 26, 129, 2012.

40. TAXAK A.K., MURUMKAR A.R., ARYA D.S. Long term spatial and temporal rainfall trends and homogeneity analysis in Wainganga basin, Central India. Weather Clim Extrem. 4, 50, 2014.

41. AHMAD I., TANG D.S., WANG M., HASHIM S. Trend analysis on precipitation time series data in munda catchment, Pakistan. Appl Mech Mater. 692, 97, 2014.

42. SHARMA C.S., PANDA S.N., PRADHAN R.P., SINGH A., KAWAMURA A. Precipitation and temperature changes in eastern India by multiple trend detection methods. Atmos Res. 180, 211, 2016.

43. WANG X., HOU X., WANG Y. Spatiotemporal variations and regional differences of extreme precipitation events in the Coastal area of China from 1961 to 2014. Atmos Res. Elsevier; 197, 94, 2017.

44. CHATTERJEE S., KHAN A., AKBARI H., WANG Y. Monotonic trends in spatio-temporal distribution and concentration of monsoon precipitation (1901-2002), West Bengal, India. Atmos Res. 182, 54, 2016.

45. ZAMAN M., YUAN S., LIU J., USMAN M., AHMAD I., CHANDIO F.A., SAIFULLAH M., LIAQAT M.U., ADNAN M. Quantifying the Effect of Climate Change on Precipitation and Temperature Patterns by Using Variant of Non-Parametric Techniques. Fresenius Environ Bull. 26, 7419, 2017.

46. ZAMAN M., FANG G., SAIFULLAH M., JAVED Q. Seasonal and Annual Precipitation Trend Prediction in Xin'Anjiang China. Fresenius Environ Bull. 25 (1), 89, 2016.

47. ZAMAN M., FANG G., MEHMOOD K., SAIFULLAH M. Trend Change Study of Climate Variables in Xin'anjiangFuchunjiang Watershed, China. Adv Meteorol. 2015, 1, 2015. 
48. YUE S., PILON P., CAVADIAS G. Power of the MannKendall and Spearman's rho tests for detecting monotonic trends in hydrological series. J Hydrol. 259, 2541, 2002.

49. KENDALL M. Rank Correlation Methods. $4^{\text {th }}$ Ed. London: Charles Griffin. 1975.

50. ZHANG Q., LIU C., XU C., XU Y., JIANG T. Observed trends of annual maximum water level and streamflow during past 130 years in the Yangtze River basin, China. J Hydrol. 324, 255, 2006.

51. GOCIC M., TRAJKOVIC S. Analysis of changes in meteorological variables using Mann-Kendall and Sen's slope estimator statistical tests in Serbia. Glob Planet Change. 100, 172, 2013.

52. BASISTHA A., ARYA D.S., GOEL N.K. Analysis of historical changes in rainfall in the Indian Himalayas. Int $\mathbf{J}$ Climatol. 29, 555, 2009.

53. AVISSAR R., WERTH D. Global hydroclimatological teleconnections resulting from tropical deforestation. J Hydrometeorol. 6, 134, 2005.

54. PIELKE R.A., ADEGOKE J., BELTRAN-PRZEKURAT A., HIEMSTRA C.A., LIN J., NAIR U.S., NIYOGI D., TIMOTHY E. An overview of regional land-use and landcover impacts on rainfall. Tellus B. 59, 587, 2007.

55. SONG Y., ACHBERGER C., LINDERHOLM H.W. Rainseason trends in precipitation and their effect in different climate regions of China during 1961-2008. Environ Res Lett. 6, 34025, 2011.

56. TIAN Y., XU Y.P., BOOIJ M.J., LIN S., ZHANG Q., LOU Z. Detection of trends in precipitation extremes in Zhejiang, east China. Theor Appl Climatol. 107, 201, 2012.

57. ZHAO P., JONES P., CAO L., YAN Z., ZHA S., ZHU Y., YU Y., TANG G. Trend of surface air temperature in Eastern China and associated large-scale climate variability over the last 100 years. J Clim. 27, 4693, 2014.

58. DONG D., HUANG G., QU X., TAO W., FAN G. Temperature trend-altitude relationship in China during 1963-2012. Theor Appl Climatol. 122, 285, 2015.

59. TANG G., DING Y., WANG S., REN G., LIU H., ZHANG L. Comparative Analysis of China Surface Air Temperature Series for the Past 100 Years. Adv Clim Chang Res. Elsevier Masson SAS, 1, 11, 2010.

60. PIAO S., CIAIS P., HUANG Y., SHEN Z., PENG S., LI J., ZHOU L., LIU H., MA Y., DING Y., FRIEDLINGSTEIN P. The impacts of climate change on water resources and agriculture in China. Nature. Nature Publishing Group. 467, 43, 2010.

61. FATHIAN F., MORID S., KAHYA E. Identification of trends in hydrological and climatic variables in Urmia Lake basin, Iran. Theor Appl Climatol. 119, 443, 2014. 